\title{
Une description des fêtes du carnaval à Brescia en l'an 1565
}

\section{Dionysios Bernicolas-Hatzopoulos}

Dans la bibliothèque Folger de Washington, D.C., est gardé un volume manuscrit, en papier, contenant la description ("relazione") d'une série de voyages en Méditerranée et en Angleterre effectués par le jeune noble vénitien Alessandro Magno quelques années avant l'occupation de Chypre par les Ottomans (1570). Le manuscrit écrit en italien sur 240 feuilles (200 x 150) s'arrête brusquement au début de la description de Cremone sur 246a (la numération de la feuille 177 est répétée deux fois). La feuille 1 est précédée de deux feuilles non numérotées dont la première porte le titre de l'ouvrage écrit à la main en italiques: Relazione del viaggio di Cipro, I di quall'isola, e di altri viaggi / fino al ritorno in Venezia di / un Patrizio veneto. / Relazione della citta e territorio / di Brescia fato dal med[esi]mo essendo / Camerlingo. Attachée à l'intérieur de l'ouverture est une note sur papier $(100 \times 120)$ portant, en cursive, l'inscription suivante: Relazione di un patrizio veneto del / viaggio di Cipro, di quell'isola ed / altri viaggi. / Relazione de medesimo della città / e territorio di Brescia. / Cod. cartaceo del sec. XVII in 4 / no 137 / Lib. di Scaf.

La feuille 246 est suivie de 37 feuilles non écrites et non numérotées. Les feuilles 59a-64b, 129b-130a, et 195, portent des numéros mais elles ne contiennent pas de texte. Le manuscrit contient plusieurs dessins en encre noire et colorée, accompagnés de légendes. Les feuilles qui en contiennent sont: f10a, désignant les vents; f68a, le système d'arosage des jardins à Chypre; f68b, maison chypriote ("forma della case di Cipro") suivi de "modo di tibiar"; f69a, "carri che usano in Cipro"; f79a, plan de la forteresse de "Sebenico"; f118b, "Volti sopra li quali è fabricata Alessandria"; f119a obélisque et "colona di Popeo"; f126a, maison égyptienne; f127a, "sala delle case al Cairo"; f130b-131a, le Sphynx et les pyramides; f132a, l'entrée et l'intérieur des pyramides; f132b, même sujet; f133b, les tombeaux des pharaons; f167b, Saint Paul de Lon- 
dres; f170a, "modo de scaricar le merci" dans le Tamise contre le courant du fleuve; f174a, moulin à vent et navire à voile; f239b, "case de i camerlenghi" et place où ont eu lieu les fêtes du carnaval de Brescia.

$\mathrm{La}$ "relazione" contient quatre voyages. Fla à f80b contiennent le premier voyage ("viaggio di Cipro"). L'auteur est resté à Chypre pendant deux ans dont quatorze mois à Pafos. Ayant quitté le port vénitien de Malamoco sur "la nave Bona nominata Santa Maria da Loreto," le 12 avril 1557, il est arrivé à Chypre vers la fin du printemps de la même année. À son arrivée il avait dixhuit ans. Il accompagnait Alessandro Zorzi, le nouveau capitaine de Pafos, qui était l'oncle de Francesco Zorzi le mari de la soeur de l'auteur, qui l'accompagnait aussi avec leur fille à son nouveau poste. Alessandro Magno est resté à Chypre jusqu'au printemps de 1559 , quand il est rentré à Venise.

La partie de son manuscrit concernant Chypre (premier voyage) contient en f17a-f58b la "relazione" sur l'île composée par Francesco Attar, "nobile Ciprio." Une partie de la "relazione" d'Attar est aussi contenue dans les "Strozzi transcripts," présentement en possession de la Bibliothèque Folger, tome 132, f119a-129a. Elle est la partie qui se réfère à l'histoire de l'île. Des extraits du mémoire de Francesco Attar ont été publiés par de Mas-Latrie qui data la rédaction du rapport vers 1540 . Évidemment la "relazione" d'Attar était bien connue et souvent utilisée, pendant l'époque de sa rédaction. Mas-Latrie, suivi plus tard par G. Hill, donne un nombre de manuscrits contenant la "relazione." 1

Les feuilles $81 a-109 b$ contiennent le deuxième voyage, encore en Chypre. Parti le 16 mars 1560 de Malamoco sur "la nave Croce di botte $900^{\prime \prime}$ il est arrivé à Pafos le 20 avril. Il était accompagné de son frère Francesco Magno. Le 23 juin 1560 les deux frères, Alessandro et Francesco, ont quitté Chypre pour arriver à Venise le 28 août 1560 .

Le troisième voyage (f110a-f146a) fut le "viaggio di Alessandria" sur "la nave Santa Maria." Parti de Venise le 4 avril 1561, le bateau est arrivé à Alexandrie le 2 mai 1561. Magno est resté en Égypte jusqu'au 19 octobre 1561 . Il est rentré à Venise le 18 novembre de la même année.

Les feuilles 146a-194b contiennent le quatrième voyage ("viaggio di Inghilterra") sur "la nave Madona Santa Maria de Loreto." Ayant quitté Venise le 1 mars 1562, les Vénitiens sont arrivés, après quelques escales de longue durée, le 7 août 1562 à Margate. Les feuilles $165 \mathrm{~b}-177 \mathrm{~b}$ contiennent des descriptions de 
Londres et des Anglais, incluant la reine Elisabeth, décrite comme une belle femme de trente ans, ainsi que certains commentaires pittoresques, comme: "le donne in tutta l'isola sono belissime" ou que la bière anglaise est "stomacosa per esser torbida come orina di cavallo."

Alessandro Magno quitta Londres le 24 septembre 1562. Arrivé à Anverse le 29 du même mois il a traversé le continent, via Cologne, Frankfort et la Suisse. Il arriva à Venise vers Noël. Il avait alors 25 ans. L'année suivante, le 27 février 1563, décide de s'établir et penser à son avenir, il fut élu "camerlengo" à Brescia. Or, les feuilles 196b-246a contiennent la description de Brescia et de sa région. Il y parle aussi de son séjour et service dans la ville. Une partie importante de ce dernier passage est occupée par une description de "giostra" à Brescia en l'an 1565 (f234a-245a), que nous publions ici à cause de son intérêt pour l'étudiant des activités urbaines dans les villes de l'Italie du nord pendant le XVIe siècle. La description de la fête par Alessandro Magno est vive et les éléments paiens de la tradition humaniste y abondent. De plus, elle est remplie d'éléments de poésie populaire où la joie de vivre déborde. Nous y sommes exposés devant une société d'un niveau de vie tres élevé, pour son temps, intellectuelle et ouverte.

Le texte est présenté tel qu'il est dans le manuscrit, à savoir sans procéder à des corrections d'orthographe à l'exception des abbréviations qui sont déchiffrées et les lettres illisibles sont remplacées par des points. Ainsi nous présentons une transcription exacte de l'original.

[234a] Li seguenti giorni sempre si hebbero passatempi diversi hora vedendo provare quelli che dovevano giostrare, et hora vedendo corer all'annello, quintana, et romper lancie, finalmente adi 25 Febraro giorno deputato ad una giostra el [cava]lier Carlo Averoldo el conte Antonio Martinengo, el conte Francesco Avogaro, et io elletti giudici dalli mantenitori alle decinove hore si riducessimo alla piazza del mercato novo, ove fra molti palchi n'era uno fatto per noi appresso el mezo della tela, et da unaltra parte in una casa si ridussero i Clar[issi]mi Rettori, ma prima ch'io passi piu oltre voglio metter qui sotto el cartello di essa giostra, et le condition sue, che sono le sottoscritte.

Per fugir l'otio cosa che porta tanto danno alla vita delli huomeni, et per dar qualche piacere con qualche virtuosa operatione in questi giorni di solazzo a questa città ma molto piu per non 
parer ingrati, et per voller pur rendere qualche guiderdone alle loro donne per le tante honeste cortesie, che ogni giorno ben aventurosamente recevono, dalla molta benignità loro, doi cavalieri servi d'Amore si sono risolti di voler provare a tutti gli innamorati, che ardirano di dir in contrario, che queste due loro donne sono le piu belle, le piu gentili, et le piu virtuose di tutte le altre di questa città, con tutto che sia piena di belle et valorose donne, la qual cosa percio credono, che non habbia bisogno di prova, perche le cose cosi chiare non si vogliono provare, pure per far la cosa ancor piu manifesta [234b] et per dar materia alli cavalieri innamorati di questa città di dimostrare o a dritto, o a torto li loro buoni animi verso le loro donne sono per provare appoggiandosi molto piu sopra la gran ragione che tengono, che sopra le forze, et valor loro quel tanto che hanno detto di sopra, pero el giorno XXV di Febraio si rittrovarano subito dopo disinare armati di tutto pezzo alla piazza del mercato novo, dove starano aspettando fino al tramontar del sole, disposti alla prova sopradetta con le conditioni, che sarano sottoscritte.

Portarano ambidoi li mantenitori duoi scudi, ne quali saranno dipinte imprese et motto, significanti gli secreti affetti delli animi loro, et quelli farano apicare nel mezo di una colonna, che per questo effetto nel loco della giostra sara piantata. Et ciascuno de venturieri similmente porti uno scudo formato nel modo sopradetto, il quale alhora che dovrà giostrare contra l'uno de mantenitori lo deponga nelle mani de s[igno]ri giudici, li quali poscia che haverano conosciuto, qual di essi si il vincitore lo faranno porre nella colonna, a sotto, o prima q[ue]llo del mantenitore secondo che il valor del cavaliere se l'havera meritato.

Et se averrà che alcuno de venturieri sia inferiore all'altro di botte, che lo suo scudo sia posto piu sotto, et parimente se egli sarà superiore che lo suo scudo sia posto piu sopra in maniera [235a] che nel fine della giostra manifestamente appaia chi piu, et meno valorosamente s'havrà portato, et se averra che doi o tre di essi siano pari, che gli scudi loro siano posti egualmente alti o bassi.

Che mentre la giostra durera niuno scudo sia levato dalla colonna, la quale poscia che sara finita ogn'uno cosi venturiero come mantenitore possa rihaver il suo, lasciando un paio di guanti al vincitore, che siano degni di lui.

Primo si ha da corere cinque carere con arme da battaglia a tutto pezzo con buffa grande senza targhetta con li pezzi doppi, che si usano nelle giostre. 
Che gli s[igno]ri giudici habbino a mettere dui confidenti uno per parte a vedere le botte, et tinte che si farano, et che niuno ardisca toccare il cavaliere fatta la carera, fin che non saranno vedute dalli signori confidenti le botte, ne possa esser nettata la tinta se prima non sarano d'accordi li s[igno]ri giudici.

Che gli s[igno]ri giudici diano dui altri confidenti, che habbino cura, di far tor le lanze rotte, et non rotte che li cavalieri correranno ogni volta, et mostrare quelle a s[igno]ri giudici, ne altri senza licenza di essi le possino mostrare, ne toccare, li quali prendino cura ancora di rivedere le lanze prima corrano i cavalieri, et farle segnare, le quali sarano tutte insieme in un loco apparecchiate a questo effetto, et da un medesmo maestro [235b] tutte fatte a un modo, da'l quale ciascuno a sua voglia le potra havere, ne si possa servir d'altre accioche la cosa vada del pari.

Che niuna lanza sia corsa piu di una volta rotta, o non rotta. Chi levera l'aversario di sella per colpo di lancia habbia otto botte, oltre la ordinaria, et guadagni quelle dello aversario, salvo se lo havesse colpito dal segno in giu, o fatto cader con un traversone, che in tal caso non si intenda haver fatto botta, ma il gettato si intenda esser fuor di giostra. Chi rompera dalla buffa in su habbia tre botte, et una e mezza toccando di punta et non rompendo.

Chi rompera dalla sonmità della buffa fin al spigolo della golla habbia due botte, et una non rompendo, ma toccando di punta.

Chi rompera da'l detto spigolo fin alla testa, o segno in su habbia una botta, et toccando di ponta, e non rompendo meza.

Chi rompera vera per vera habbia due botte et chi incontrara non rompendo ne habbia una.

Chi rompera nella schiffa habbia due botte, et non rompendo ma toccando di punta una, avertendo che se uno toccasse in far le sopraditte botte di punta, et poi rompesse di traversone si intenda haver fatto tinta e non rotta.

Chi si lasciara cascar la lanza perdi una botta, o chi dara della lanza su le tele, ma non pero siano a guadagno dell' aversario [236a] et se ben facesse botta dopo dato su le tele sia nulla.

Che se per diffetto di tempo non si potesse corere quel giorno, o finire sia rimesso al di seguente, o come meglio parera a s[igno]ri giudici a quali sia rimessa ancora ogn'altra difficultà che potesse occorere. Queste adonque erano le conditioni della giostra, a veder la quale riddutisi li signor Rettori, et noi giudici all'hora soprascritta alle venti hore comparsero li dui mantenitori con questo ordine venivano prima quattro trombetti dui d'essi vestidi di veste aquartade di color bianco et argentino, et li altri dui di bianco et 
pavonazzo, driedo essi dui moretti sopra bellissimi cavalli vestiti di veste intagiade con habito moresco, quali portavano le piche, et scudi delli cavalieri con le loro imprese, seguivano poi li cavalieri uno d'essi ch'era el cavalier Provai chiamato Tristan de Leonis coperto lui et il cavalo con sopravesta fatta a intagli di [bereteni: mot omis sur lequel est tirée une ligne] argentino sopra bianco con bellissimo penon di penacchi dello istesso colore con uno bastone in mano. L'altro ch'era el s[ign]or Riciardo Avogadro chiamato Amadis di Gaula era allo istesso modo vestito, ma in loco di argentino havea color pavonazzo, et erano circondati da quattro staffieri vestiti della insegna e colore delli loro patroni. Venivano driedo essi li lor patrini con forse 60 gentilhuomeni parte d'essi con una posta argentina con cordelina d'argento attraversata dalle spale, et ligata sotto il braccio manco, et parte con poste pavonazze et bianche. [236b] E con questo ordine poi c'hebbero fatta la mostra attorno la piazza si fermorno ad un de capi della tela, ove erano stesi dui paviglioni et si assisero alla porta d'uno d'essi, facendo appendere li loro scudi ad una colonna ch'era appresso li loro paviglioni con tre man di gradi a modo di aguglia. Haveva Amadis di Gaula per impresa nel suo scudo. Due navi in fortuna di mare, una d'esse somersa, e l'altra in modo di salvarsi con la luce di sant'Ermo in gabbia, (che dopo gran fortuna da speranza di salute alli navicanti.) con moto Propitii luminis benignitate.

Tristano de Leonis haveva nel suo. Una fiera con testa di serpe con denti lunghi a modo di Elefante, e con orecchie lunghe pendenti con collo lungo e sottile, con squame d'oro, di color rosso, verde, et altri insieme. Il corpo grosso e pieno, le gambe corte con piedi a guisa d'occhi et è di tal vaghezza che stando al sole risplende talmente che gli augelli si fermano a riguardarlo con moto. Sasselo Amor.

Non stete molto dapoi el comparir delli mantenitori che gionsero in piazza dui aventurieri con honorata comp[agni]a ma senza livrea, quali mentre era il popolo intento a riguardare dui altri cavalieri venuti soli senza comp[agni]a di niuno da due diverse parti, gionti l'uno da una [237a] parte della tela e dall'altra l'altro improvisamente si mossero l'uno contra l'altro, e rotte valorosamente le lancie tratti li stocchi si ferivano gagliardamente, quando dubbioso ogn'uno del fine, ne sapendo perche fusse la loro battaglia sopragionse uno vestito di donna a cavalo, e postosi fra loro li rappacifici insieme, e con essi venuto al nostro cospetto ne presentò queste due seguenti stantie. 


\begin{abstract}
Questi doi franchi e forti cavaglieri
Havean per me tra lor presa battaglia.

Desiando ciascun de mei pensieri

Farsi signor vestirno piastra e maglia,

E con animi invitti, arditi, e altieri

Cercan mostrar qual piu di loro vaglia.

A questa festa anch'io ridotta a caso

Differir lor tenzon gl'ho persuaso.

Non perche ogni gentil alma cortese

sol ha de la virtu cura, e desio

Ciascun d'un par voler l'alma s'accese

Ma molto piu per il consiglio mio.

Di provar hoggi in tanto degne impresse

Quanto puo l'arte et il valor natio

Percio signori giusti almi et elletti

Non dispreggiate i loro honesti affeti
\end{abstract}

Driedo essi comparse una compagnia di quindeci nimfe sopra [237b] cavali bianchi, et vestite di bianco traendo ovi d'aqua rose, driedo le qual veniva Diana con un cavaliero seco al pari vestita al modo istesso che le nimfe, et il cavaliero era vestito di argento e bianco, con un capeletto di tela d'argento in testa, et il suo cavallo era coperto dello istesso di che era la sua sopravesta, et inanzi d'esso havea uno a cavallo che li portava l'elmo, e lo scudo, e quattro staffieri vestiti anch'essi di bianco, et attorniata la piazza s'appressorno a noi, e Diana ne presentò la sottoscritta scrittura.

Diana alli M[agnifi]ci e prudentissimi s[igno]ri.

Era talmente adirato il coro celeste per la cecità de certi, i quali cosi cecamente caminano nelle cose terrene, che benche habbiano occhi sono totalmente di lume privi che ne neggano ne giudichino se non al senso, che alcuni de i Dei erano (merce della discordia) per abbandonare il cielo, e suscitare unaltra fiata il sommo Jove, il quale unaltra volta haverebbe fatto tristi Encelado et Tifeo, o che sarebbe ita un'altra fiata la Gretia tutta alla rovina di Troia. Ma io havendo cio previsto per menor male sono fra gli homini rittornata, da'l comercio de quali per mia honestade erami alle solitarie selve ritirata, et de quanti che delle cose divine si dilletano da'l senso alieni, et che hanno lo spirito alle virtu celesti elevato, ho scielto et qui meco menato questo virtuoso, honesto, et [238a] valoroso cavaliero, il quale talmente diffendera (cosi spero) la comune honestade offesa, che nissuno, il quale delle cose honeste si dilletti restara da lui offeso. Questo giudicarete con il sano, et intero giudicio vostro voi Minoi et Radamanti.

Dopo questo ne comparsero dui altri, e perche piu non ne haveano a venir el s[ign]or Hipolito Albrico cognominato el cavalier della Croce che era comparso primo ne presentò il suo scudo, nel qual era depinto un cavaliero armato e legato con catena di ferro, 
che era tenuta per mano di una dongella con moto spagnolo, che dice: Asi mas gozo siando atado, que no quando era librado. Et era vestito senza pompa niuna, e preso del campo, e corse le cinque carere che dovea con Tristano de Leonis rimase perdente, et mandassimo a metter il suo scudo sotto l'altro del vincitore.

Presentò il suo scudo poi el s[ign]or Claudio Peschiera detto el cavalier dalla speranza, nel qual era figurato uno scoglio in mezo el mare percosso dall'onde con motto Conantia frangere frango. Et era coperto lui, et il cavallo di coperta e sopravesta violada e bianca con penacchiera dello istesso colore, et postosi da un capo della tela contra se li mose Amadis, et rimase inferior di [238b] meza botta, onde il suo scudo fu posto di sotto a quello del suo aversario cioè di Amadis.

Driedo esso fu presentato el scudo del s[ign]or Cesareo Averoldo chiamato el cavalier di Diana che havea per impresa un'aguila che si leva a volo con due talpe ne i piedi, e motto che dice: Maiora molior, et corso le quattro carere per esser l'hora tarda si mise fine, et fu bandita la giostra per el giorno seguente, nel quale raddunatisi tutti alli 19 hore, corse el sopraditto cavaliero l'altra carera, et rimase vinto da Tristano, et il suo scudo fu posto al disotto.

Segui el s[ign]or Thomaso Pelusella detto el cavalier incognito coperto di chermesino, e portava nello scudo: Una donzella con un freno in mano in atto di doglia con motto: Ai que la pena mi manda dezir, i la ragion callar. Et rimase vinto da Amadis.

El s[ign]or Scipion Provai poi coperto di gialo si presentò con impresa di una scova, o spazzadora con doi cavalieri entro, che fanno la seda con motto che dice: Meus labor aliorum comodum, et si addimandava el cavalier travaglioso, et fu inferior di botte a Tristano.

Venne a lui driedo el s[ign]or Camillo Soraga detto el cavalier della fortuna, portando per insegna uno Asparago con [239a] motto: Sic semper denuo renascor, et in tanto fu fortunato che ottene vittoria, vincendo Amadis.

Ne a lui hebbe invidia el s[ign]or Marco Tullio Bargnano detto el cavalier dalla luce, che havea nel scudo un sole, et una luna alquanto piu bassa con moto: Inde lux. percioche anch'esso vinse Tristano. Non vi restando altri a corere insieme co'l giorno finì anco la giostra, e li mantenitori triomfanti con gran rumor di tamburi, e suon di trombe accompagnati da gran moltitudine di gentilhuomeni con li scudi inanzi se ne andarono alle case loro. El Marti si giostrò all'anello, et si ruppero lancie nella istessa piazza. 
El mercore nel palazzo del Clar[issi]mo Podesta el s[ign]or Bortolamio Arvigio lettor dell'Accademia bressana lesse quel sonetto del Petrarca che dice: Liete, pensose, accompagnate, $e$ sole, et in essa lettione molto si diffuse a ragionar della gelosia esortando le donne (percioche ve n'erano molte, e dongelle, e maritate, e vedove) con molti essempi a chiuder gli occhi, e non dar mente alle operationi del marito, et finita che fu si ballò fino alle tre hore di notte. El giovedi nella cità non si fece cosa niuna, percioche tutti escono fuora a veder diverse feste, che si fanno su la spianata, et la città fino alle ventitre hore riman si puo dir [239b] vota tanto poche persone si veggono in essa, e perche non possano tutti doi li rettori star fuori ad un tratto, essendo uscito el Clar[issi]mo Podesta feci compagnia al Clar[issi]mo Cap[itan]o cavalcando attorno la terra, e guardando dalle murra li molti cocchi, e carette, che si vedevano fuori per le strade. El venerdi si giostrò alla quintana. El sabato si combatè la barera piovendo sempre, che fece riuscir la festa men bella di quello che dovea essere pur fu bellissima, e volendola narar è necessario descriver el loco che è la piazza ove sono le nostre case, a similitudine di questo disegno [La moitié de la page 239b est occupée par le dessin]. [240a] nell qual inanzi la tore, che è alta come ogni gran campanile et si chiama del populo fabricorno una prospettiva bellissima, dandoli nome di tempio dedicato a Venere, et inanzi la porta di esso vi fecero uno ponte, per il quale si discendeva da'l tempio nel stecato, dalle bande del quale vi'erano molte haste con lumiere, che ardevano sempre et sopra il tempio vi erano cinque piramide, che nella cima havevano alcune balle dorate, due delle qual, che erano dalli canti erano ripiene di fuochi artificiati, che di continuo gittorno varii fuochi da'l cominciar della festa fino alla fine, e fra esse vi erano alcuni vasi, che sempre rendetero una grande et bellissima luce, cosa che faceva bellissimo veder. Ne era men bello il veder il giorno li molti palchi che coprivano tutta la piazza fabricati tre e quattro uno sopra l'altro, et coperti di tavole.

Ma vedendo alla festa nello imbrunir della sera venero li s[ign]ori Rettori sopra el loro palco fabricato inanzi le nostre case, et poco dapoi el s[ign]or Lucretio da Gambara maestro del campo con la ponpa, et ordine come qui sotto. Prima havea inanzi di se otto huomini vestiti alla Albanesa come usano li stratioti con veste lunghe a quartieri bianchi e neri, con capelli in testa pur bianchi e neri, con scimitare a lato, et questi portavano dui torci per uno, uno inanzi e l'altro gettato sopra la spala rendeva lume di driedo, e fra loro andavano quattro e sei gentilhuomeni con alabarde, se- 
guivano quattro trombetti a cavalo vestiti allo istesso modo, ma con veste piu [240b] corte, poi un piciol nano vestito medesimamente, et driedo lui el maestro del campo sopra uno bellissimo cavalo in colletto di brocato d'oro, et bragoni dello istesso con una posta nera al colo con cordeline d'oro attorno, et uno capelletto in testa circondato da dodeci alabardieri, et quattro servitori con torcie con cape nere intorno listate di veluto bianco, lo seguivano altri gentilhuomeni con altri otto servitori vestidi, et con torci come li primi. Venero driedo essi fino al numero di cento fra soldati et alambardieri armati, quali essendo stati ordenati dalli s[ign]ori Rettori accio non nascesse alcun disordine si posero fra una e l'altra palificata a guardia del stecato, e similmente di servitori con le torcie, et li gentilhuomeni si divisero attorno el stecato. Poco dapoi la gionta di questi si videro nella tore molti fuochi, e si uditero sonar trombette, et si vide uscir fuori una cassa con doi cavalieri armati entro quale ardeva per tutto intorno, et di essa da varie parti ne uscivano diversi fuochi et schioppi, et a poco a poco vene a basso fra la tore, et la prospettiva del tempio, dalla qual usciti li cavalieri et venuti nello steccato armati di bellissime arme, e con bellissimi cimieri (in loco del quale uno haveva uno idolo inanzi el quale vi ardeva un piciol foco con viva fiamma) fatta la mostra s'assisero alla porta del tempio, espettando che li venturieri comparissero, quali uno driedo l'altro fino al numero di quindeci con diverse bellissime inventioni e con molte torcie poco dapoi comparsero, quale tratto da dongella, qual da anemale, alcuni legati da Cupido, et altri [241a] ad altro modo con diverse livree, fra quali ne comparsero tre vestiti da remiti con uno Orfeo inanzi che con due stantie, che cantò nella lira mostrò che essendosi rettirati dalle arme, et dattosi alla vita solitaria havendo inteso che doi si vantavano non si rittrovar piu leali di loro in Amore ripprese le arme voleano dimostrarli, che mantenevano il falso, et tratto l'habito Eremitico rimasero in arme bianche, comparsi questi se udi dar una parte della piazza trar due coete, al . . . delle quali da'l nostro palco si levò uno vestito da Mercurio, che andava gettando alcuni cartelli, per li quali si vedeva, che Giove veduta le presontion delli mantenitori, e sapendo non vi esser piu al mondo huomo che chiamar si possa valoroso mandava Mercurio all'interno per trarne de li dui di quelli cavalieri antichi, e condurli a combatter con quelli e da'l palco andò a terra, e dato del caduceo, ove nel dissegno si vede il pozzo (qual era stato levato et coperto, che parea [ . . . . . ] fusse stato cosa alcuna) et in esso per una casa li vicina, et per una cava che sotterra fecero vi entravano, et usci- 
vano subito levate le tavole, et terra, che vi era sopra si ap[pres]se una paventosa e gran lesta di crudelissima fiera, che rassomigliava propriamente la bocca dello inferno secondo che vien depinta et era tanto grande quanto capir poteva la larghezza del pozzo, et furno sbarate due altre coete, et di essa (che gittava rochette et fuoco dalla bocca, da gli occhi, da'l naso, et da ogni parte) ne usciva Cerbaro cane trifauce gettando fuoco anch'esso, et si volse contra Mercurio, qual dattoli del Caduceo sopra l'un de capi se lo stese [241b] a piedi, e cade nella fossa, o bocca, qual tuttavia gettava fuoco da ogni parte. Poco stete che vi uscirono forse dieci diavoli con diverse maniere de fuochi chi portandolo nelle mani, chi nella coda, chi nel capo, et altri in altra maniera, facendosi molto bene dar luoco alle genti, furno seguiti questi da altri venti con due torcie nere per cadauno, et poi venero li dui cavalieri con sopraveste nere, quale strassinavano per terra, et penoni bellissimi similmente neri, et fatta la lor mostra attorno el stecato, percioche piu non ne doveano venire cominciorno lo abattimento, principiando quelli che primi comparsero, e seguendo li altri per ordine, el modo del qual conbattimento fu che stando uno delli mantenitori da una parte della barera, et uno delli venturieri dall'altra erano portate sei piche tre delle quali ne ellegeva lo venturiero, e l'altre tre rimanevano allo mantenitore, e similmente due lancie una era a sua elletta del venturiero, et l'altra del mantenitore, quale si rompevano adosso, et del tronco della lancia si davano una botta alla testa, poi tratti li stochi si ferivano anco con essi fino che erano separati da'l M[aest]ro del campo quale dava al vincitore un fior di seta, che era portato dallo adversario per premio del vincitore. Mentre si combatteva mai cessorno li fuochi del tempio, e la bocca anco dell'inferno sempre gettava fuoco e broncie grossissime fino alla fine, che poi si chiuse, e la barera anch'essa arse di diversi fuochi cosa bellissima da veder se la pioggia non la havesse sturbata un poco. Li Mantenitori erano el Conte Alfonso Capriolo, quale alla seconda volta che combatè si fece [242a] male ad una mano, et per lui combatè con li altri tutti, che fu el S[ign]or Ferante Averoldo, et durò la festa fino alle hore cinque di notte, et vi erano trecento e sessanta torcie, quali con la luce loro di notte facevano giorno.

La domenica fu pioggia, e le donne non sapendo che farsi venivano a veder uno castello fatto con molto artificio, che era nell'altra casa de i Camerlenghi, nel qual si vedevano al girar solo di una ruota lavorar in un tempo istesso come se fussero vivi diverse arte, dar la batteria al castello, sbarar le artiglierie, quelli di dentro 
diffendersi, andar la guardia attorno, et far altri varii effetti, oltra cio uno horologio far li istessi effetti con li 3 Magi, et suonar di hore come quello di S. Marco in Venetia, et meglio, et due fontane gettar sempre aqua abondantemente senza spander mai fuori del vaso con altri molti bellissimi misteri.

El Lunedi le nimfe che comparsero alla giostra con il cavaliero di Diana giostrorno all/anello un pretio di scudi quaranta.

El Marti non si potendo far festa fuori della cita (come era usanza) per la pioggia ne fecero per la città in casa di diversi gentilhuomeni, ond'io che molti giorni havea aspettato occasione et non ne havea habbuto il comodo, vedendo che il Carnovale era alla fine deliberai di mascherarmi, et trovati altri sei compagni, et tolti cavali a nolo, vestitisi da cavalari, over corieri con buoni stivali, et feltri intorno, et vestito uno servitore da bagaglione sonando il corno sempre corressimo la posta per tutta la città, poi fermatisi a diver- [242b] se feste, dispensassimo li sottoscritti terzetti, quali composi io come meglio me parea che convenissero a quelli a chi erano drecciati, e chiusi a modo di [......] con la mansion sopra fingevamo che venissero di Fiandra et $[\ldots \ldots$. . . alli S[ign]or Rettori, ma questo non hebbe ricapito perche non uscirono delli loro Palaggi.

Vostro saggio governo almi signori

Rissuona tanto in queste nostre parte,

Che molti saggi spinti in voce, $\mathrm{e}^{\prime} \mathrm{n}$ carte

Si son messi a cantar de vostri honori.

Et io che son fra lor de li minori

Vo raccogliendo l'alte vostre parte,

Ne trovo con qual via, ne con qual arte

Possin lodarvi a pieno altri scrittori.

Felice Brescia, e piu felice ancora

Gli cittadini suoi poi che ci è dato.

D'administrar giustitia a i stati loro.

Che'l saper vostro qual mostrate ogn'hora

A quello bene aventurato stato

Rittornera la prima eta dell'oro.

Alla S[igno]ra Podestaressa.

Unqua donna vid'io piu di voi degna

Di cottal grado e di cotesto honore

In cui somm'honesta e prudenza regna.

Al S[ign]or Conte Camillo Calin. 
Superfluo è a voi Calini haver la scala.

[243a] Che tanto ascende l'alto nome vostro

Quanto ascender potria se havesse l'ala.

Percioche hano la scala nell'arma.

Alla S[igno]ra Vittoria sua consorte.

Fra Palade e Junon la tua bellezza

A Venere assimiglia vaga Dea,

Che rende alla citta gloria e vaghezza.

Volendo intendere per Palade e Junon due sue cognate, l'una la S[igno]ra Barbara vedova e sorella del marito molto savia e l'altra la S[igno]ra Lavinia consorte del Conte Pietro.

Al S[ign]or Conte Pietro Calin.

Hai moglie non curar se ben si dice.

Che inutil sei nel feminil comercio

Che ben san molti s'hai buona radice.

Questo da se è chiaro.

Alla S[igno]ra Lavinia sua consorte.

Godi senza temer felice sposa,

Ch'altri t'inudi il tuo signor benigno.

Poi che ad ognun la verita è nascosa.

Al S[ign]or Pietro Martinengo.

Mal puo sua nobiltade un arbor spandere, Che di se un sol germoglio produtto habbia, $C^{\prime}$ ha seco mille rischi, e si puo frangere.

Ha questo gentilhuomo un figlio solo et è vedovo.

[243b] Al S[ign]or Ricciardo Avogadro.

Aventuroso cavalier d'Amore.

Ti doni il Dio cupido ampia vittoria,

Che dell'amata goda a tutte l'hore,

Era innamorato di una gentildonna delle Magi ricche. 
Al cavalier Carlo Averoldo.

Non opera di streghe o magica arte,

Di che tanto ti duole signor mio,

Ma il tempo hormai da te il vigor diparte,

Perche diceva esser legato e percio non poter usar piu con donne, il che veniva da vecchiezza.

Al S[ign]or Alouise Martinengo.

Senza te che amo sempre et ama ancora, Et è per amar piu di giorno in giorno

Non fara tua moglier lunga dimora,

Perche era gelosa di lui et esso credeva partir per Cipro.

Alla S[igno]ra Lelia sua consorte.

Se voi sola ama e apprezza il car consorte,

Vostra felicita non veggo cosa

Che disturbar la possi altro che morte.

Alla S[igno]ra Claudia Martinenga vedova.

Come vite senz'arbor non s'apprèzza,

E mal sta sola ogni tenera pianta

Cosi senz'huomo sta vostra bellezza.

Alla S[igno]ra Barbara Calina.

Barbaro è il nome tuo nobil signora, [244a] Ma tal è la virtude e l'honestade

Che non pur Brescia, ma il mondo ti honora.

Al S[ign]or Zuan-Maria Peschiera.

De delicati cibi, pesci, e frutti

V'è mirabil peschiera, e si cortese,

Che da se ne fa copia e dona a tutti,

Percioche tien sempre corteggiato li regimenti di diverse gentilezze.

Al S[ign]or Conte Alfonso Capriolo. 
Corre la fama tua gentil Capriolo

A guisa del cognome e si s'inalza

Che poco piu faria se andasse a volo.

Alla S[igno]ra Hortensia sua consorte.

Non diro che da cruda gelosia,

Ma da sincero amor che tu li porti,

Temi che'l tuo signor tolto ti sia.

Al S[ign]or Conte Marc'Ant[oni]o Martinengo.

Al braccio hai il ceppo, al colo la cattena

E questo accio si vegga manifesto

Che la tua diva a sua voglia ti mena.

Alla S[igno]ra Paula sua consorte.

Chi vol veder unita una beltade

Voi sola guardi, voi sola contempli

Piena di real costumi e di honestade.

Al S[ign]or Conte Camillo Martinengo.

Quel gran furio Camillo quel Romano, Che sottopose a Roma tanti imperi

Ceda al novo Camillo Bresciano.

E molto altero.

Alla S[igno]ra Polissena consorte del S[ign]or Camilo Mart[inen]go.

Bella, gentil, cortese, honesta, e pura

Quanto fu donna mai saggia et acorta

Per farvi pose el signor ogni cura.

Al S[ign]or Camilo Avogaro.

Greve è a mie spale e poderosa soma, Voler tue lodi dir saggio Camillo Che tal se a Bressa qual quell'altro a Roma. 
Al S[ign]or Conte Antonio Martinengo.

Invidia sol ha tolto il degno grado A tua virtu e sapienza citta non solo

A governar una citta, ma un stado,

Perche era cascato in uno certo officio, che dà la citta.

Al S[ign]or Julio Martinengo.

El grave andar e l'altero sembiante

El signoril vestir la faccia reggia

Fa che merti ad ognun andar inante,

Serva gran gravita nello andare.

Alla S[igno]ra Grandilia sua consorte.

Gratie, che a pochi el ciel largo destina

Rara virtu, bellezza, e leggiadria,

Fa che ogn'huomo v'adora e vi s'inchina.

Al S[ign]or Conte Ruberto Avogadro.

Prudentia e governarsi con modestia

E non ceder all ira facilmente,

Che spesso po[r]ge al huom danno e modestia.

Questo Conte si [245a] incontro li giorni passati appresso el palazzo del S[ign[or Cap[itan]o con el Conte Scipion Porcelaga, ove per vecchia inimicitia fra loro non voleano dar loco uno all'altro, onde li servitori messero mano all'arme, essi non mai, ma ne volevano tornar adrieto come erano pregati da diversi gentilhuomeni, ne darsi loco fino che giongendo el S[ign]or Cap[itan]o da una parte, et io dall' altra li conducessimo in driedo.

Alla S[igno]ra Paula Gonzaga.

Li tuoi belli occhi e'l delicato viso

La bionda chioma, e'l gratioso andare

Apre a sua voglia, e chiude'l paradiso.

Al Conte Lelio Martinengo. 
Di bella e nobil donna accompagnato

Di eta viril, robusto, ricco, e saggio

Godi felice e aventuroso stato.

Al S[ign]or Conte Fr[ancesc]o Avogadro.

Che dolce piu, che piu giocondo stato Seria di quel di uno amoroso core Quando come voi fusse aventurato.

Alla S[igno]ra Laura Averolda.

Tanto sei donna fra le belle bella, $\mathrm{E}$ si riluce il tuo gentil aspetto

Come riluce il sol piu di ogni stella.

Al S[ign[or Claudio Peschiera.

Fra questa generosa e nobil schiera

Di valerosi e gentil cavalieri,

Pochi a te s'appareggiano Peschiera.

Alla S[igno]ra Orontea sua consorte.

Mentre leggiadramente vi volgete

Danzando ogniuno in voi stupido mira

Come smella et a tempo vi movete

[le mot volgete est effacé dans le texte par une ligne, et remplacé par movete].

Alla S[igno]ra Cavaliera Calina.

Gentil e vaga donna qual virtute

Debbo piu in te lodar! che molte sono,

E molto anco da molti conosciute!

Alla contessa Lionora Martinenga.

Di Magnanimita di splendor reggio

Altiera donna sei la principale

Che merta fama, gloria, honor, e preggio. 
Et con questa mascherata finirono le feste et il carnovale con uno grande contento. Havevamo anco ogni domenica di sera fino alle sei hore gran passatempo, percioche s'erimo convenutti dodeci fra di camera, et Capitani de soldati, et posta ogniuno la sua portione dapoi diversi giochi e passatempi cenavimo in comp[agni]a alli quali a tutte mie spese convitando anco el Clar[issi]mo Cap[itan]o el giovedi grasso diedi una cena et li feci un festino di putte artegiane con le quali passassimo allegramente la maggior parte della notte, e cosi havessimo un Carnovale godevole.

Université de Montréal

\section{NOTE}

1. M.L. de Mas-Latrie, Histoire de l'ile de Chypre sous le regne des princes de la maison de Lusignan, III (Paris, 1855), p. 493, n. 1, pp. 519-36, Documents, voir aussi, p. 519, n. 1; G. Hill, A History of Cyprus, III, The Frankish Period, 1432-1571 (Cambridge, 1948) p. 765, n. 1. 